\section{Sensibilización sobre los objetivos de desarrollo sostenible a través de repentinas de diseño de sistemas de producto-servicio.}

Marco Ferruzca, Alinne Sánchez Paredes y

Carolina Andrade ${ }^{(1)}$

\begin{abstract}
Resumen: El presente trabajo da testimonio de la experiencia de una escuela de diseño realizando talleres de diseño orientados a promover los objetivos de desarrollo sostenible (ODS), así como proponer sistemas de producto-servicio para alcanzar dichos objetivos. $\mathrm{Al}$ mismo tiempo que los participantes se familiarizan con los ODS, aprenden a usar nuevas herramientas que favorecen la creatividad para diseñar sistemas de producto-servicio. La experiencia de cuatro años organizando este tipo de experiencias ha conducido a proponer el diseño de un nuevo curso que se integra al plan de estudios de las licenciaturas que ofrece la escuela en cuestión.
\end{abstract}

Palabras clave: Diseño sostenible - Objetivos de Desarrollo Sostenible - SSPS - Diseño de servicios.

[Resúmenes en inglés y portugués en la página 69]

(1) División de Ciencias y Artes para el Diseño, Universidad Autónoma Metropolitana, México.

\title{
Introducción
}

La agenda 2030 para alcanzar los objetivos del desarrollo sostenible fue lanzada en 2015, poniendo énfasis en que en el año de 2020 se deberían dinamizar durante la próxima década acciones desarrolladas e implementadas por organizaciones de educación en todos los niveles, organizaciones de la sociedad civil, organizaciones privadas y públicas, y ciudadanía; sin embargo, la pandemia mundial ha traído cambios sustanciales y ha acelerado otros, como la pobreza. De acuerdo al Fondo Monetario Internacional, la economía mundial se enfrenta ahora a su peor recesión desde la Gran Depresión, y Oxfam Internacional ha advertido que 500 millones de personas podrían verse empujadas a la pobreza derivado de la crisis sanitaria.

Es debido a lo anterior que la importancia de desarrollar acciones para alcanzar los objetivos 2030 es imperante, haciendo énfasis en la construcción de una sociedad más equi- 
tativa, inclusiva, sostenible, y la sociedad en su conjunto pueda gozar de bienestar en su vida diaria.

\section{Objetivos de Desarrollo Sostenible}

El mundo enfrenta grandes desafíos que ponen en riesgo su desarrollo sostenible. Esta situación reconocida por diferentes líderes mundiales en 2015 dio como resultado la creación de una agenda para el 2030 orientada a eliminar la pobreza, salvaguardar el planeta, mejorar el acceso a la educación, entre otros objetivos. En total se reconocen 17 objetivos de desarrollo sostenible (ODS) en los que la sociedad en su conjunto debería trabajar para contribuir a reducir brechas que en los últimos años se han hecho más evidentes: fin de la pobreza, hambre cero, salud y bienestar, educación de calidad, igualdad de género, agua limpia y saneamiento, energía asequible y no contaminante, trabajo decente y crecimiento económico, industria-innovación-infraestructura, reducción de las desigualdades, ciudades y comunidades sostenibles, producción y consumo responsable, acción por el clima, vida submarina, vida de ecosistemas terrestres, paz, justicia e instituciones sólidas, y finalmente, alianzas para lograr los objetivos (Naciones Unidas, 2015).

$\mathrm{Al}$ respecto, las instituciones de educación superior deben asumir un papel importante para alcanzar dichos objetivos, en virtud de sus capacidades para transformar a la sociedad, a través de sus funciones sustantivas como son la docencia, investigación, preservación y difusión de la cultura. No obstante, deberán de experimentar un cambio al interior de su propia cultura organizativa para perfilar un nuevo modelo de universidad para la sostenibilidad (Instituto Internacional de la UNESCO para la Educación Superior en América Latina y el Caribe, 2020).

Con el fin de impulsar la agenda en las universidades para alcanzar los ODS, la Red de Soluciones para el Desarrollo Sostenible (SDSN, por sus siglas en inglés), publicaron la guía "Como empezar con los ODS en las universidades" (SDSN Australia / Pácifico, 2018). De este documento se desprenden algunas acciones como:

- Proporcionar conocimientos a docentes y alumnos para entender los ODS.

- Desarrollar habilidades en el alumnado que permitan abordar los desafíos de los ODS

- Impulsar proyectos de investigación orientados a brindar soluciones a los ODS

- Alinear el aparato estructural y legislativo de las universidades con los ODS

\section{Diseño de sistema producto-servicio}

El concepto de sistema producto-servicio ha sido ampliamente estudiado, sin embargo, en la última década ha cobrado mucha atención dentro del dominio disciplinar del diseño (Viveros et al., 2019). Una manera sencilla de explicar este concepto es como un conjunto de productos y servicios capaces de atender y satisfacer las necesidades de los usuarios. 
En virtud del papel fundamental de las y los diseñadores como promotores de la innovación en las organizaciones, es relevante que desarrollen un pensamiento estratégico que contribuya a la construcción colaborativa de un conjunto de productos, servicios o estrategias de comunicación que un actor o conjunto de actores conciben y desarrollan para obtener resultados estratégicos.

La disciplina del diseño dispone de un conjunto de conocimientos, metodologías y métodos útiles para el diseño de este tipo de sistemas que puede estar orientado a alcanzar los ODS.

\section{Repentinas de diseño ODS}

El presente caso hace una descripción general de los talleres organizados durante los últimos cuatro años por una escuela de diseño para promover el conocimiento de los ODS en el alumnado, así como para desarrollar habilidades que les permitan abordarlos desde la óptica del diseño de sistemas producto-servicio. Los talleres han seguido la propuesta metodológica proporcionada por la iniciativa Global Goals Jam (GGJ) creada en 2016 (Digital Society School, 2020) y consisten en una jornada intensiva de dos días, que en este trabajo denominamos repentinas, en la que los participantes colaboran en la búsqueda de soluciones a problemas locales vinculados a los ODS. Los talleres procuran realizarse al mismo tiempo en las diferentes sedes alrededor del mundo con el fin de tener un encuentro entre pares a nivel global. El carácter multidisciplinario entre creativos, investigadores y expertos es fundamental para obtener mejores soluciones. Como parte de los GGJ se utilizan herramientas que guían el proceso de diseño, con énfasis en el trabajo colaborativo (Digital Society School, 2020). El caso se organiza en cinco apartados: identificación de la situación, diseño del estudio de caso, conducción del estudio de caso, análisis de resultados y conclusiones.

\section{Identificación de la situación}

Desde el año 2017 se han organizado de manera anual los talleres de GGJ con la colaboración de varios docentes que han fungido como mentores en un principio. Sin embargo, en los últimos dos años, alumnos que han participado en las primeras ediciones de los GGJ se han sumado como mentores también. La temática de los ODS no era parte de la currícula de ninguno de los planes y programas de estudio ofrecidos en la escuela.

\section{Diseño del caso}

La realización de este estudio es de corte descriptivo y servirá para responder a la siguiente pregunta:

¿Qué podemos aprender de introducir el conocimiento de los ODS a través de repentinas de diseño?

Para responder a esta pregunta se identificarán materiales que sirvan para el análisis y discusión posterior. La unidad de análisis son los talleres en sí mismo. 


\section{Conducción del caso}

La evidencia de propuestas de sistemas de producto-servicio para alcanzar los ODS se recogió a partir de los materiales elaborados por los alumnos durante los talleres y otros generados dentro de la propia escuela.

Al tratarse de una experiencia ex post facto no hubo selección de participantes, pero esto sirve como una base para responder la pregunta anteriormente planteada en el diseño del estudio. La tabla No 1 muestra los datos obtenidos.

Tabla 1. Relación de materiales analizados.

\begin{tabular}{lll}
\hline Descripción & Tipo & Observaciones \\
\hline $\begin{array}{l}\text { GGJ (Experiencia de los } \\
\text { participantes) }\end{array}$ & $\begin{array}{l}\text { Preguntas abiertas sobre su } \\
\text { experiencia de participación en los } \\
\text { talleres }\end{array}$ & Participantes y Organizadores \\
\hline GGJ (Resultados de proyectos) & $\begin{array}{l}\text { Prototipos conceptuales de } \\
\text { soluciones que contribuyen a } \\
\text { alcanzar los ODS. }\end{array}$ & Participantes y Organizadores \\
\hline $\begin{array}{l}\text { Diseñar para el desarrollo } \\
\text { sostenible }\end{array}$ & Programa de estudio & Creadores \\
\hline
\end{tabular}

\section{Análisis}

La posibilidad de organizar y participar en varias de las iniciativas mencionadas en la tabla 1 , permiten identificar algunos factores vinculados al interés por parte de alumnos y profesores respecto a los ODS. La tabla 2 presenta una síntesis de esos hallazgos y los factores a los que están asociados.

Tabla 2. Relación de hallazgos y factores asociados.

\begin{tabular}{ll}
\hline Factores & Objetivos de Desarrollo Sostenible y Diseño \\
\hline El compromiso con la & Como parte de la retroalimentación que se ha recibido de los GGJ, destaca el \\
sociedad & reconocimiento de los participantes por: - compartir experiencias y preocupaciones \\
& sobre problemas comunes como la violencia de género, la falta de vivienda digna, la \\
& falta de inclusión en las ciudades, entre otros. \\
& - resaltar la importancia del diseño en proyectos sociales. \\
& - buscar un bien común. \\
& - la posibilidad de tener encuentros de trabajo colaborativo globales con otras \\
& personas en el mundo con problemas similares. \\
& Así mismo, al final de las actividades, los participantes reconocen la sensación de no \\
& perder la esperanza por tener un mejor planeta y se motivan para superarse. \\
\hline
\end{tabular}




\begin{tabular}{ll}
\hline Actualización de & Todos los invitados participantes se mostraron interesados en las actividades, \\
conocimientos & y reconocen haber identificado nuevas formas de plantear o enfrentar problemas \\
& sociales. Destaca la oportunidad de proyectar de una forma novedosa soluciones a \\
& problemas más complejos.
\end{tabular}

Desarrollo de nuevas habilidades
Los participantes declaran estar satisfechos con la forma colaborativa de llevar a cabo los talleres porque les permite trabajar con otras disciplinas, incluir a otros actores de la sociedad, como usuarios y público en general, el gobierno y el sector productivo.

Formación

Si bien los talleres GGJ han servido como un instrumento para que los alumnos diseñen productos-servicios orientados a enfrentar los ODS, la escuela ha hecho un esfuerzo para crear un curso denominado "Diseñar para el desarrollo sostenible" cuyo objetivo general es entender y analizar la importancia del campo del diseño para alcanzar los objetivos de desarrollo sostenible. Se trata de un curso teórico-práctico de 4.5 horas a la semana con equivalencia en créditos. El curso es interdisciplinario y permite la inscripción de arquitectos, diseñadores de la comunicación gráfica y diseñadores industriales. Consta de 12 semanas. De esta manera, se ha introducido de manera formal los ODS en los planes y programas de estudio.

Otros aspectos relevantes La adecuación reciente (2020) a los planes de estudio de la licenciatura en Diseño Industrial abrió una oportunidad para incluir en el objetivo de la carrera lo siguiente: "Formar un profesional integrado, con una conciencia crítica de su actividad disciplinaria y de la relación existente entre éste y la sociedad; que conozca racionalmente, mediante su proceso de diseño de productos, servicios, estrategias, y experiencias; el compromiso, el enfoque, las funciones, aportaciones y la conciencia con la cual se diseña y realiza un producto socialmente útil."

\section{A continuación se muestran algunas fotografías de los talleres:}

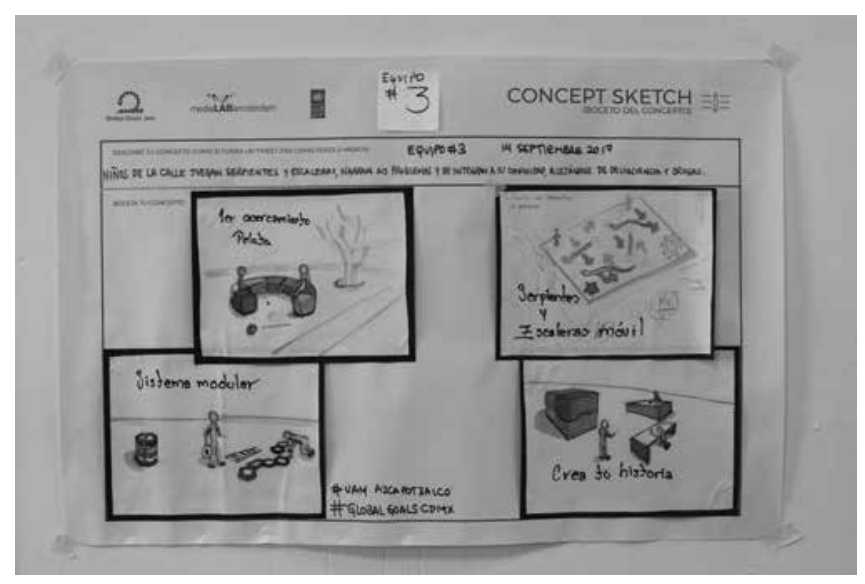

Figura 1. Propuestas conceptuales de diseño para promover la integración social. 

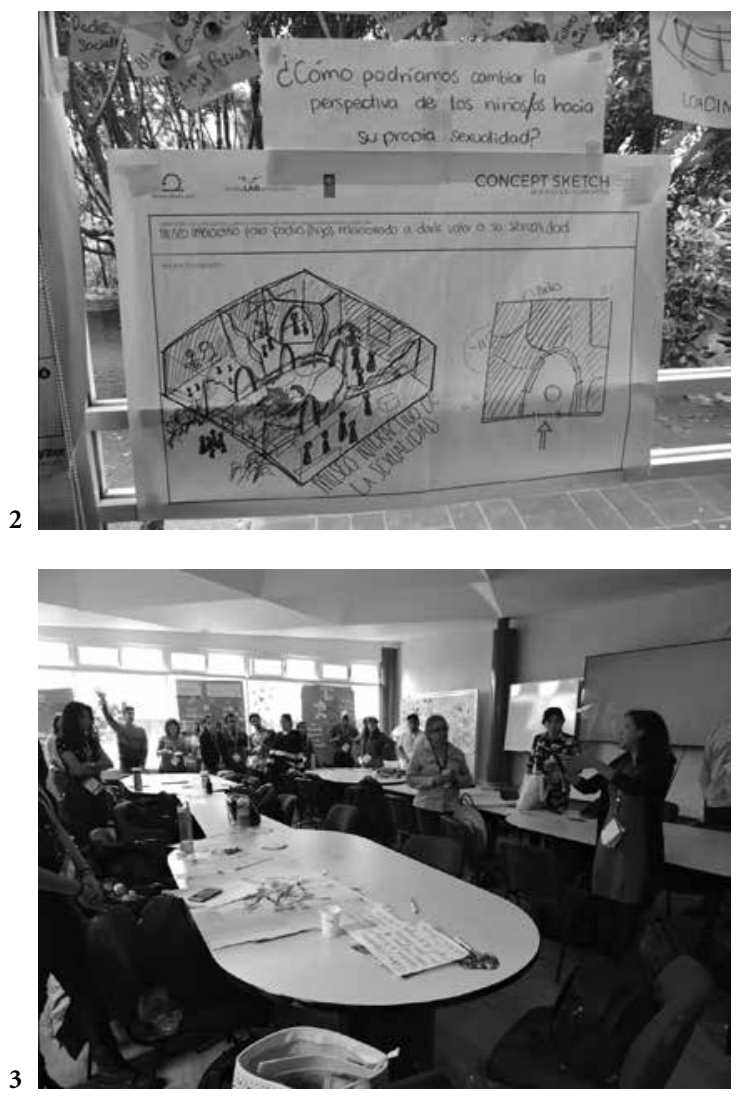

Figura 2. Propuesta conceptual de un museo interactivo de la sexualidad.

Figura 3. Trabajo colaborativo entre expertas, alumnos y profesores.

\section{Reporte del caso}

\section{a. El caso o el problema}

Como se ha podido ver en este caso, la introducción de los ODS como un tema relevante para ampliar el conocimiento de los diseñadores se ha realizado a través de talleres y de la actualización del plan de estudio. La retroalimentación obtenida nos permite afirmar que el alumnado está interesado en estos temas y por lo tanto deseoso de contribuir a buscar soluciones. De igual manera, se muestra abierto a trabajar en equipos multidisciplinarios y emplear nuevas formas de proyectar sistemas de productos-servicios.

En síntesis, la posibilidad de organizar pequeñas repentinas sobre los ODS, ha contribuido mejor a entender cómo plantear la temática dentro de los planes y programas de estudio de diseño. 
b. Consideraciones para elaborar el caso

La posibilidad de los autores para realizar los talleres ODS bajo la metodología de los GGJ ha sido fundamental para familiarizarse con la temática, descubrir nuevos conocimientos y aplicarlos al interior de la escuela. Es una manera muy práctica de aproximarse a los retos que enfrenta la sociedad. Los talleres GGJ son fáciles de implementar en las escuelas de diseño porque estamos familiarizados con muchas de las herramientas que proponen y sobretodo por centrar el enfoque de estudio en las personas.

c. Resultados

La posibilidad de involucrarse en todos los talleres como organizadores, ha servido para tener una mejor idea sobre la oportunidad que representa para del diseñador crear sistemas de producto-servicio con una orientación más social. Asimismo, han servido para impulsar una adecuación a los planes de estudios que en su objetivo de formación incluye el estudio de los objetos de desarrollo sostenible y la generación práctica de soluciones.

d. Retos y cómo se enfrentaron

Uno de los retos que se presentaron, fue entrenar a profesores que no estaban familiarizados con las herramientas de los GGJ. Sin embargo, como ya se mencionó, este tipo de herramientas están basadas en la actividad proyectual, por lo que para los diseñadores son fáciles de asimilar. Se organizaron sesiones de entrenamiento con los profesores que fungieron como mentores.

\section{Implicaciones relevantes para el campo de estudio}

Una actividad como la que realizan los diseñadores, requiere ser más consciente tanto en su saber como en su hacer. El diseño es una parte muy importante de la cultura material que produce el ser humano, así como de su comportamiento con todo lo que nos rodea. Por ejemplo, los hábitos de producción y consumo de las personas deben cambiar porque es insostenible el ritmo al que lo hacemos. Esto supone para los diseñadores repensar nuevas formas de consumir y producir. De igual manera, los diseñadores tienen una responsabilidad al momento de proyectar mensajes, objetos o espacios incluyentes, por lo que además de desarrollar un pensamiento creativo, también deben desarrollar un pensamiento integral orientado por el desarrollo sostenible.

Como escuelas superiores de diseño debemos proporcionar los conocimientos que permitan a los alumnos y docentes entender mejor los ODS, y desarrollar una forma de pensamiento sistémica que nos ayude a contribuir de la mejor forma a resolver los problemas que aquejan a la sociedad.

\section{Conclusiones}

El mundo actual está sometido a diversas fuerzas que están transformando la manera en que vivimos, nos movemos, nos comportamos, etc. A saber, la producción y la manufactura avanzada, los materiales avanzados, la robótica, la biotecnología, las comunicaciones 
digitales, los drones, etc. Para el diseño es de suma importancia seguir de cerca estas grandes transformaciones porque generarán nuevos sistemas de producto-servicio para los usuarios, nuevos comportamientos de consumo y nuevas formas de interactuar.

Desde la perspectiva docente, es fundamental que los futuros diseñadores tengan conocimiento de los ODS, con el objetivo de incorporar su profesionalización aspectos globales y locales para coadyuvar en el desarrollo de la sociedad

Desde la perspectiva de escuela de diseño, es necesario incorporar en las funciones sustantivas (docencia, investigación, preservación de la cultura) acciones encaminadas al desarrollo sostenible. En el caso de la UAM-Azcapotzalco en la facultad de diseño, se ha buscado desde el año 2017 incorporar cursos, acciones y reforzar en los planes y programas de estudio de licenciatura y posgrado como eje transversal el desarrollo sostenible.

La generación de políticas públicas en torno a la divulgación, creación y fortalecimiento de acciones para alcanzar el Desarrollo Sostenible 2030, permitirá tener las bases para el crecimiento y desarrollo económico del país, que puede traducirse en mejorar la calidad de vida de sus habitantes.

La elaboración de este artículo se da inmerso en la pandemia por Covid-19, que ha traído cambios nodales en la forma en que nos desenvolvemos cotidianamente, como es el trabajo, la escuela, la economía, entre otros; estos cambios de acuerdo al Fondo Monetario Internacional, la economía mundial se enfrenta ahora a su peor recesión desde la Gran Depresión, y millones de personas podrían verse empujadas a la pobreza derivado de la crisis sanitaria, por lo que la explosión de servicios digitales en diferentes ámbitos abre una oportunidad para que el diseño como disciplina asuma su papel en esta nueva normalidad y contribuya a mejorar la necesidad de los usuarios a través de la interacción con mensajes, objetos y espacios, sean estos físicos o virtuales.

\section{Agradecimiento}

Un especial agradecimiento a Valeria Valdez por toda su colaboración en los últimos cuatros años durante la realización de los GGJ.

\section{Referencias}

Digital Society School. (2020, Abril 28). Global Goals Jam. Global Goals Jam. https:// globalgoalsjam.org/about/

Digital Society School. (2020). Design method toolkit using the toolkit. Resources - Digital Society School. https://toolkits.dss.cloud/design/using-the-toolkit/

Instituto Internacional de la UNESCO para la Educación Superior en América Latina y el Caribe. (2020). Contribución de la educación superior a los objetivos de desarrollo sostenible: marco analítico. UNESCO-IESALC. http://www.iesalc.unesco.org 
Organización de las Naciones Unidas (2020). Informe de los Objetivos de Desarrollo Sostenible 2020. https://unstats.un.org/sdgs/report/2020/The-Sustainable-Development -Goals-Report-2020_Spanish.pdf

Naciones Unidas. (2015, septiembre 2). A/70/1 - S - A/70/1. eSubscription to United Nations Documents. https://undocs.org/es/A/70/1

SDSN Australia /Pacífico. (2018, marzo 1). Disponible en español la Guía "Como empezar con los ODS en las universidades”. Red Española para el Desarrollo Sostenible. https:// reds-sdsn.es/guia-empezar-los-ods-las-universidades

Viveros, B. A.; Öhrwall-Rönnbäck, A.; Ferruzca, M. V. \& Ramírez, A. (2019). The ISPIM Innovation Conference - Celebrating Innovation: 500 Years Since daVinci. International Society for Professional Innovation Management. https://search.proquest.com/openview/ d5be79cbd17a7e2c41aef33902932ce3/1.pdf?cbl=1796422\&loginDisplay=true\&pq-orig site $=$ gscholar

\begin{abstract}
This paper testifies to the experience of a design school conducting design workshops aimed at promoting sustainable development goals (SDG), as well as proposing product-service systems to achieve these goals. At the same time that the participants become familiar with the SDGs, they learn to use new tools that promote creativity to design product-service systems. The experience of four years organizing this type of experience has led to the proposal of the design of a new course that is integrated into the curriculum of the bachelor's degrees offered by the school in question.
\end{abstract}

Keywords: Sustainable design - Sustainable Development Goals - SSPS - Service design.

Resumo: Este artigo atesta a experiência de uma escola de design conduzindo workshops de design com o objetivo de promover objetivos de desenvolvimento sustentável (ODS), bem como propor sistemas de produto-serviço para atingir esses objetivos. Ao mesmo tempo que os participantes se familiarizam com os ODS, eles aprendem a usar novas ferramentas que promovem a criatividade para projetar sistemas produto-serviço. A experiência de quatro anos organizando este tipo de experiência levou à proposta de desenho de um novo curso que se integre no currículo dos cursos de licenciatura oferecidos pela escola em questão.

Palavras chave: Design sustentável - Objetivos de Desenvolvimento Sustentável - SSPS Design de serviço.

[Las traducciones de los abstracts fueron supervisadas por el autor de cada artículo] 